\title{
Visceral adipose tissue in patients with COVID-19: risk stratification for severity
}

\author{
Hersh Chandarana ${ }^{1}$ (D) $\cdot$ Bari Dane ${ }^{1} \cdot$ Artem Mikheev $^{1} \cdot$ Myles T. Taffel $^{1} \cdot$ Yang Feng $^{2} \cdot$ Henry Rusinek $^{1}$
}

Received: 8 June 2020 / Revised: 23 July 2020 / Accepted: 25 July 2020 / Published online: 3 August 2020

๑) Springer Science+Business Media, LLC, part of Springer Nature 2020

\begin{abstract}
Purpose To assess visceral (VAT), subcutaneous (SAT), and total adipose tissue (TAT) estimates at abdominopelvic CT in COVID-19 patients with different severity, and analyze Body Mass Index (BMI) and CT estimates of fat content in patients requiring hospitalization.

Methods In this retrospective IRB approved HIPPA compliant study, 51 patients with SARS-CoV-2 infection with abdominopelvic CT were included. Patients were stratified based on disease severity as outpatient (no hospital admission) and patients who were hospitalized. Subset of hospitalized patient required mechanical ventilation (MV). A radiologist blinded to the clinical outcome evaluated single axial slice on $\mathrm{CT}$ at $\mathrm{L} 3$ vertebral body for $\mathrm{VAT}_{\mathrm{L} 3}, \mathrm{SAT}_{\mathrm{L} 3}, \mathrm{TAT}_{\mathrm{L} 3}$, and $\mathrm{VAT}_{\mathrm{TAT}}$. $\mathrm{These}$ measures along with age, gender, and BMI were compared. A clinical model that included age, sex, and BMI was compared to clinical $+\mathrm{CT}$ model that also included $\mathrm{VAT}_{\mathrm{L} 3}$ to discriminate hospitalized patients from outpatients.

Results There were ten outpatients and 41 hospitalized patients. 11 hospitalized patients required MV. There were no significant differences in age and BMI between the hospitalized and outpatients (all $p>0.05$ ). There was significantly higher $\mathrm{VAT}_{\mathrm{L} 3}$ and $\mathrm{VAT} / \mathrm{TAT}_{\mathrm{L} 3}$ in hospitalized patients compared to the outpatients (all $p<0.05$ ). Area under the curve (AUC) of the clinical +CT model was higher compared to the clinical model (AUC 0.847 versus 0.750 ) for identifying patients requiring hospitalization.

Conclusion Higher $\mathrm{VAT}_{\mathrm{L} 3}$ was observed in COVID-19 patients that required hospitalization compared to the outpatients, and addition of $\mathrm{VAT}_{\mathrm{L} 3}$ to the clinical model improved $\mathrm{AUC}$ in discriminating hospitalized from outpatients in this preliminary study.
\end{abstract}

Keywords Visceral adipose tissue $(\mathrm{VAT}) \cdot \mathrm{CT} \cdot \mathrm{COVID}-19$

\section{Introduction}

A new RNA betacoronavirus called severe acute respiratory syndrome coronavirus 2 (SARS-CoV-2) is responsible for the illness COVID-19 [1-3] that has resulted in, and continues to cause severe global morbidity and mortality. COVID-19 was classified as a global pandemic by the World

Hersh Chandarana

Hersh.Chandarana@nyulangone.org

1 Center for Advanced Imaging Innovation and Research (CAI2R), and Bernard and Irene Schwartz Center for Biomedical Imaging, Department of Radiology, New York University Grossman School of Medicine, 660 First Ave, New York, NY 10016, USA

2 Department of Biostatistics, School of Global Public Health, New York University, New York, NY, USA
Health Organization (WHO) on March 11, 2020. There are two major hypothesized mechanisms for viral infection related injury: (1) the virus binds to Angiotensin Converting Enzyme 2 (ACE2) human cell receptor which is highly expressed in type 2 alveolar cells, vascular epithelium, and cardiac myocytes; (2) a cytokine storm related to an exuberant pro-inflammatory state [4]. The spectrum of symptomatic infection ranges from mild to critical illness. Some patients with initially mild symptoms may progress over the course of a week and develop more severe disease, which may require mechanical ventilation. Pneumonia is one of the common manifestation of lung injury. Acute respiratory distress syndrome (ARDS) requiring invasive mechanical ventilation $(\mathrm{MV})$ is a major pulmonary complication in patients with severe disease and can manifest shortly after the onset of dyspnea. Therefore, there is a strong need to identify and validate biomarkers that are associated with 
severe symptoms and worse prognosis. Reliable prediction of high-risk patient phenotype can allow us to optimize resources and closely monitor those patients who are likely to require hospitalization, intensive care unit (ICU) admission, and MV.

Recent studies have identified a number of risk factors that predispose patients to severe illness. These include age, male sex, diabetes mellitus, hypertension, cardiopulmonary diseases, and obesity [5-7]. A number of studies have identified obesity as a risk factor not only for hospitalization but also for MV requirement in patients with COVID-19 [8]. Obesity is also associated with an increased risk of metabolic disorders such as diabetes mellitus, and cardiovascular diseases [9]. Obesity is considered a state of low-grade inflammation [10], with various inflammatory products secreted by adipose tissue. The main adipose tissue-derived inflammatory cytokines include TNFa, IL-1, and IL-6. Recent reports suggest an increased inflammatory environment leading to cytokine storm in significant number of patients with COVID-19 [11]. The mechanism of viral infection and the development of obesity seem to share some common metabolic and inflammatory reaction pathways. Infection of individuals who are already showing increased inflammation may result in higher morbidity. This could potentially provide an explanation as to why obesity is associated with worse outcome [12].

All fat depot in the body are not created equal; they confer different cardiovascular and metabolic risks [13, 14]. Subcutaneous adipose tissue (SAT) is relatively indolent, whereas visceral adipose tissue (VAT) is metabolically more active tissue that secretes inflammatory cytokines [14]. VAT is linked to metabolic syndrome, cardiovascular impairment, and increased susceptibility to infection and sepsis [15]. Hence, it is of interest to analyze the relationship between the amount of visceral fat and the severity of COVID-19 infection. VAT and SAT can be measured and classified rapidly and accurately using MRI or CT [16]. While it is possible to quantify the amount of adipose tissue within the whole abdomen, several studies have demonstrated that the single-slice measurement made rapidly at the vertebral body $\mathrm{L} 3$ or $\mathrm{L} 4$ level is an excellent estimate of the abdominal VAT and SAT in both men and women and across the spectrum of obesity $[17,18]$.

The aim of this study was twofold: (1) to assess VAT, SAT, and total adipose tissue (TAT) on abdominopelvic CT in SARS-CoV-2-positive patients with different severity of COVID-19 infection, and (2) to analyze body mass index (BMI) and fat content estimated at $\mathrm{CT}$ in patients with COVID-19 who required hospitalization.

\section{Methods}

\section{Subjects}

This study was approved by the institutional review board and was HIPAA compliant and received waiver of informed consent. A retrospective institutional database search identified all adults ( $\geq 18$ years of age) who had an abdominopelvic CT from March 19, 2020 through April 19, 2020 with "COVID" in the CT report. This search initially identified 72 patients. Eighteen patients were excluded as COVID-19 positivity was not confirmed by the review of the electronic medical record (EMR). This resulted in the study cohort of 54 patients (38 men and 16 women; mean $[ \pm \mathrm{SD}]$ age, 59.8 [14.9] years; age range 20-88 years). PCR-positive diagnosis of SARS-CoV-2 infection was confirmed in all 54 patients. Indications for the CT were abdominal pain $(n=26)$, fever $(n=5)$, sepsis/hypotension $(n=4)$, acute kidney injury $(n=2)$, cancer $(n=2)$, GI bleed $(n=2)$, trauma $(n=3)$, poor oral intake/SBO/diarrhea $(n=5)$, vascular indications $(n=3)$, abnormal liver function test $(n=1)$, and hematuria $(n=1)$. Three patients were excluded due to unavailability of axial imaging for analysis $(n=1)$, and presence of extensive ascites $(n=2)$ which precludes evaluation of visceral fat (Fig. 1). The EMR was reviewed to categorize patients into different phenotypic cohorts based on disease severity: (1) patients that did not require hospital admission (outpatient), (2) patients who were hospitalized (hospitalized), and (3) subset of hospitalized patient who were admitted to the ICU and required invasive MV (hospitalized $+\mathrm{MV}$ ). Of the 10 outpatients, 6 patients presented to ER/urgent care and 4 patients presented to outpatient imaging facility. Age, gender, and Body Mass Index (BMI) were also collected in all patients when available (Table 1). BMI was unavailable in three patients.

Some of these patients were included in two other imaging studies that focused on qualitative imaging findings at lung bases and findings of hypercoagulability in COVID19 patients [19].

\section{CT segmentation of VAT and SAT}

De-identified axial DICOM CT images were parsed and loaded to the NIH supported FireVoxel software (wp.nyu. edu/firevoxel). Axial images were reformatted into sagittal (Fig. 2a) and coronal projections from which the slice corresponding to the superior endplate of the L3 vertebral body was identified. Quantitative adipose tissue analysis at L3 abdominal level was performed by a board certified abdominal radiologist with 12 years of the post-fellowship experience. The reader was blinded to the clinical outcome. 


\section{2 adult patients with abdominopelvic CT exam between March \\ 19 and April 19 with report that included term 'COVID'}

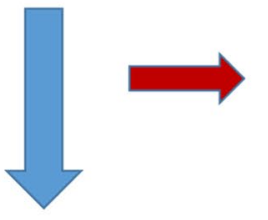

\section{8 patients excluded as COVID-19 diagnosis was not confirmed with PCR test}

\section{4 adult patients with PCR positive diagnosis of SARS-CoV-2 infection and CT exam}

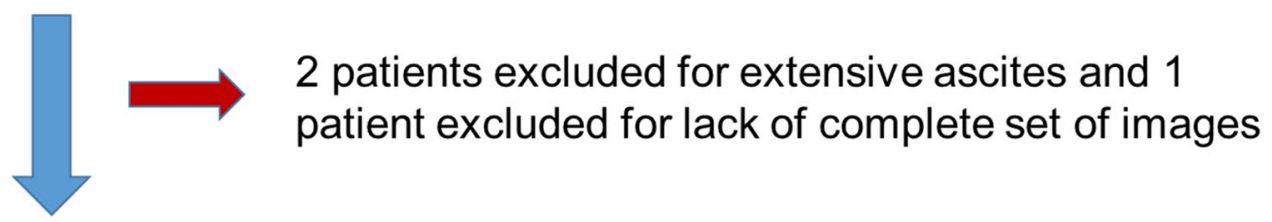

\section{1 adult patients included in the final cohort}

Fig. 1 Patient inclusion and exclusion criteria for study cohort of patients with PCR diagnosis of SARS-CoV-2 infection and abdominopelvic CT examination performed between March 19 and April 19, 2020

Table 1 Demographics of patients with COVID-19

\begin{tabular}{llll}
\hline & Outpatient & $\begin{array}{l}\text { Hospitalized } \\
\text { but no MV }\end{array}$ & Hospitalized + MV \\
\hline Number of subjects & 10 & 30 & 11 \\
Age & $54.7 \pm 11.6$ & $58.2 \pm 17$ & $67.9 \pm 10.4$ \\
Sex & $5 \mathrm{M}, 5 \mathrm{~F}$ & $22 \mathrm{M}, 8 \mathrm{~F}$ & $11 \mathrm{M}, 0 \mathrm{~F}$ \\
BMI $\left(\mathrm{kg} / \mathrm{m}^{2}\right)$ & $28.1 \pm 7.6$ & $30.4 \pm 7.8$ & $27.6 \pm 2.3$ \\
\hline
\end{tabular}

The semi-automated analysis includes the following steps:

(1) Body masking was performed automatically to remove all outside objects unrelated to the abdomen such as the supporting table, patient arms, and tubing.

(2) Automatic median filtering, followed by thresholding to [-120, - 90] Hounsfield Units (HU) range, and morphological noise removal yielded the total fat (TAT) mask (Fig. 2b).

(3) The abdominal cavity contour was generated by placing between 10 and 20 anchor points (mouse clicks) along the margin of the peritoneal surface of the abdominal cavity (Fig. 2c).

(4) TAT voxels were automatically partitioned in two classes: those inside the abdominal cavity contour were labeled as VAT, and those outside as SAT (Fig. 2d). The total areas of SAT $\left(\mathrm{SAT}_{\mathrm{L} 3}\right)$, VAT $\left(\mathrm{VAT}_{\mathrm{L} 3}\right)$ and TAT $\left(\mathrm{TAT}_{\mathrm{L} 3}\right)$ in $\mathrm{cm}^{2}$ were computed.

To verify measurement repeatability, another observer (over 25 years of experience in image processing) independently calculated L3-level visceral and subcutaneous fat measures on a randomly selected subset of 25 cases as described above.

To test correlation between single-slice and volumetric measurements, another independent observer performed a full 3D segmentation, from the level of the diaphragm to the sacrum, on a randomly sampled subset. The step (3) in analysis pipeline described above was performed on every fifth slices and automatically interpolated in z-direction. The remaining steps for computing $\mathrm{VAT}_{3 \mathrm{D}}$, and $\mathrm{SAT}_{3 \mathrm{D}}$ were as for the single-slice analysis. All 3D measurements were done by a reader blinded to the clinical outcome as well as singleslice analysis.

\section{Statistical analysis}

Single-slice (at the L3 vertebral body level) $\mathrm{VAT}_{\mathrm{L} 3}, \mathrm{SAT}_{\mathrm{L} 3}$, $\mathrm{TAT}_{\mathrm{L} 3}, \mathrm{VAT} / \mathrm{TAT}_{\mathrm{L} 3}$, and BMI were compared using Mann-Whitney test for independent samples between the outpatient and hospitalized patients, as well as in the hospitalized patients that did not require MV versus those that were admitted to the ICU and required MV (hospitalized $+\mathrm{MV})$. These data were also stratified by gender. $\mathrm{VAT}_{\mathrm{L} 3}, \mathrm{SAT}_{\mathrm{L} 3}, \mathrm{TAT}_{\mathrm{L} 3}$, and $\mathrm{VAT}_{\text {TAT }} \mathrm{T}_{3}$ measures were correlated with BMI. The intraclass correlation coefficient (absolute agreement version) was used to assess repeatability in $\mathrm{VAT}_{\mathrm{L} 3}, \mathrm{SAT}_{\mathrm{L} 3}$, and $\mathrm{VAT} \mathrm{TAT}_{\mathrm{L} 3}$ in 25 subjects with measurement providers by two independent observers. Volumetric measure of $\mathrm{VAT}_{3 \mathrm{D}}, \mathrm{SAT}_{3 \mathrm{D}}$, and $\mathrm{VAT} / \mathrm{TAT}_{3 \mathrm{D}}$ were correlated to single-slice L3 measurements (in $\mathrm{cm}^{2}$ ) in 25 subjects. 
a

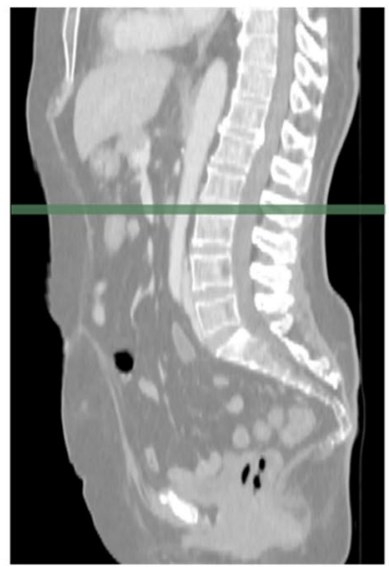

C

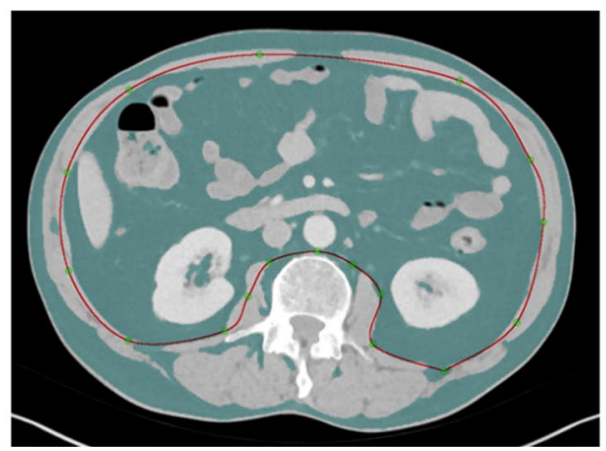

Fig. 2 a Selection of the axial slice at the superior end plate of L3 vertebral body. b Histogram of attenuation values within selected CT slice shows a well-defined peak with the maximum at -110 Hounsfield Unit (HU). c Axial slice with superimposed teal color overlay for voxels in $[-120,-90] \mathrm{HU}$ attenuation range. The expert observer

In the next step, two logistic regression models were fitted to discriminate patients requiring hospitalization from those that were outpatients. A clinical model that included age, sex, and BMI was compared to a clinical + CT model that also included $\mathrm{VAT}_{\mathrm{L} 3}$. The two models were compared with BIC and Chi-squared test. ROC analysis was performed and area under the curve (AUC) was computed for both of the models to discriminate patients requiring hospitalization from outpatients.

MedCalc (version 19.2.6) and $\mathrm{R}$ (version 3.6.3) were used to perform statistical analysis.

\section{Results}

Ten patients with a diagnosis of COVID-19 were not admitted to the hospital (outpatient), whereas the remaining 41 patients were hospitalized. Of these 41 hospitalized patients, 11 patients were admitted to the ICU and required MV, and b

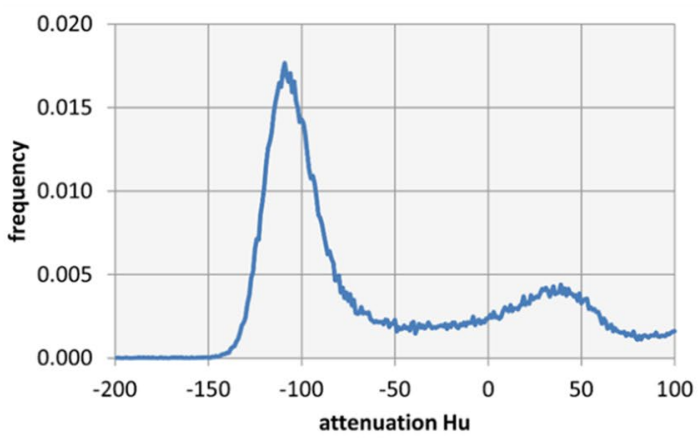

d

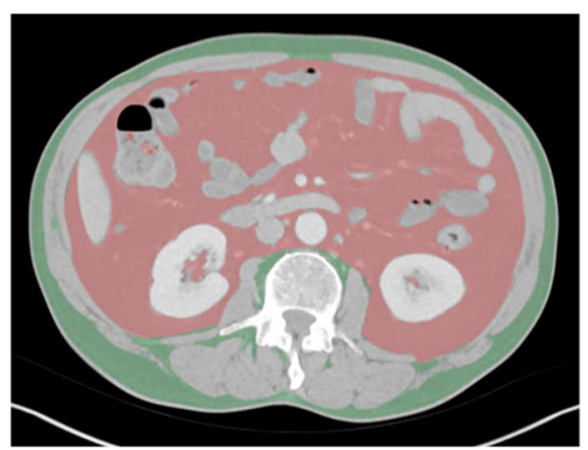

partitioned the mask by placing a contour of approximately 20 anchor points (thin red line) to outline abdominal cavity. d Fat voxels inside the abdominal cavity contour were labeled as VAT (red) and fat voxels outside the abdominal cavity were identified as SAT (green)

the remaining 30 hospitalized patients were not admitted to the ICU.

\section{Age}

The mean age of all the patients was $59.8 \pm 14.9$ years. The age of the outpatients $(n=10)$ was $54.7 \pm 11.6$ years and the age of all the hospitalized patients $(n=41)$ was $60.8 \pm 15.8$ years. There was no significant difference between the outpatient and the hospitalized patients $(p>0.05)$. Patients requiring MV and ICU admission were older with age of $67.9 \pm 10.4$ years. Although patients requiring $\mathrm{MV}$ and ICU admission were older compared to other hospitalized and outpatients this did not reach statistical significance. 


\section{Gender}

There were a total of 13 women and 38 men. $50 \%$ of outpatients were men $(n=5)$, whereas $80 \%$ of all the hospitalized patients were men $(n=33)$. Furthermore, all the ICU patients were men $(n=10)$. This difference between the outpatient and hospitalized patients was statistically significant $(p=0.047)$.

\section{BMI}

The BMI in the outpatient and hospitalized patients were not significantly different $\left(28.1 \pm 7.6 \mathrm{~kg} / \mathrm{m}^{2}\right.$ vs. $29.8 \pm 6.7 \mathrm{~kg} /$ $\mathrm{m}^{2}$ ). Furthermore, there were no differences in BMI of the ICU patients $\left(27.6 \pm 2.3 \mathrm{~kg} / \mathrm{m}^{2}\right)$ versus hospitalized patients. All $p$ values $>0.05$.

\section{$\operatorname{VAT}_{L 3}$ and $S_{A T}$}

The $\mathrm{VAT}_{\mathrm{L} 3}, \mathrm{SAT}_{\mathrm{L} 3}, \mathrm{TAT}_{\mathrm{L} 3}$, and $\mathrm{VAT}_{\mathrm{TAT}}$ in in the three phenotypic cohorts based on disease severity are shown

Table $2 \mathrm{VAT}_{\mathrm{L} 3}, \mathrm{SAT}_{\mathrm{L} 3}, \mathrm{TAT}_{\mathrm{L} 3}$, and VAT/TAT $\mathrm{L}$ in the three phenotypic cohorts based on disease severity

\begin{tabular}{lccl}
\hline & Outpatient & $\begin{array}{l}\text { Hospitalized but } \\
\text { no MV }\end{array}$ & Hospitalized + MV \\
\hline VAT $\left(\mathrm{cm}^{2}\right)$ & $128.0 \pm 92.1$ & $224.2 \pm 115.9$ & $240.6 \pm 101.2$ \\
SAT $\left(\mathrm{cm}^{2}\right)$ & $232.3 \pm 125.3$ & $231.5 \pm 142.2$ & $179.6 \pm 56$ \\
TAT $\left(\mathrm{cm}^{2}\right)$ & $360.4 \pm 149.1$ & $455.7 \pm 201.0$ & $420.2 \pm 134.7$ \\
VAT/TAT & $0.35 \pm 0.2$ & $0.50 \pm 0.16$ & $0.56 \pm 0.08$ \\
\hline
\end{tabular}

There was a significant difference in $\mathrm{VAT}_{\mathrm{L} 3}$ and $\mathrm{VAT} / \mathrm{TAT}_{\mathrm{L} 3}$ in the hospitalized patients when compared to the outpatients $(p=0.01)$. There were no significant differences in $\mathrm{SAT}_{\mathrm{L}_{3}}$ and $\mathrm{TAT}_{\mathrm{L} 3}$ between the hospitalized patients and the outpatients (all $p>0.5$ ) in Table 2. There was a significant difference in $\mathrm{VAT}_{\mathrm{L3}}$ and VAT/TAT $\mathrm{L}_{3}$ in the hospitalized patients when compared to the outpatients $\left(\mathrm{VAT}_{\mathrm{L} 3}: 228.6 \pm 111.1 \mathrm{~cm}^{2}\right.$ vs. $128.0 \pm 92.1 \mathrm{~cm}^{2}, p=0.01 ;$ and VAT/TAT $\mathrm{L}_{3}: 0.52 \pm 0.14$ vs. $0.35 \pm 0.20, p=0.01$ ); Fig. 3 . There were no significant differences in $\mathrm{SAT}_{\mathrm{L} 3}$ and $\mathrm{TAT}_{\mathrm{L} 3}$ between the hospitalized patients and the outpatients (all $p>0.5$ ).

$\mathrm{VAT}_{\mathrm{L} 3}$ in the patients on MV in the ICU was higher than in the hospitalized patients not requiring MV, but this was not statistically significant (Table 2). There were no significant differences in $\mathrm{SAT}_{\mathrm{L} 3}, \mathrm{TAT}_{\mathrm{L} 3}$, and $\mathrm{VAT} / \mathrm{TAT}_{\mathrm{L} 3}$ between patients requiring $\mathrm{MV}$ and hospitalized patients that did not require $\mathrm{MV}$ (all $p>0.05$ ).

\section{Comparison stratified by gender}

Women: There were five female outpatients and eight hospitalized female patients. The $\mathrm{VAT}_{\mathrm{L} 3}$ in hospitalized female patients was significantly higher than outpatients $\left(175.8 \pm 55.5 \mathrm{~cm}^{2}\right.$ vs. $\left.92.4 \pm 54.1 \mathrm{~cm}^{2} ; p=0.03\right)$ (Table 3$)$.

Men: There were five outpatient men and 33 hospitalized men. The mean $\mathrm{VAT}_{\mathrm{L} 3}, \mathrm{TAT}_{\mathrm{L} 3}$, and $\mathrm{VAT} \mathrm{TAT}_{\mathrm{L} 3}$ were higher in hospitalized men compared to the outpatient men (Table 4), but these differences did not reach statistical significance (all $p>0.05$ ).

Table $3 \mathrm{VAT}_{\mathrm{L} 3}, \mathrm{SAT}_{\mathrm{L} 3}, \mathrm{TAT}_{\mathrm{L} 3}$, and $\mathrm{VAT} / \mathrm{TAT}_{\mathrm{L} 3}$ in outpatients and hospitalized women

\begin{tabular}{lrcr}
\hline & \multicolumn{1}{c}{ Outpatient } & Hospitalized & $p$ value \\
\hline VAT $\left(\mathrm{cm}^{2}\right)$ & $92.4 \pm 54.1$ & $175.8 \pm 55.5$ & 0.03 \\
SAT $\left(\mathrm{cm}^{2}\right)$ & $238.0 \pm 83.6$ & $308.8 \pm 126.2$ & $>0.05$ \\
TAT $\left(\mathrm{cm}^{2}\right)$ & $330.4 \pm 81.7$ & $484.6 \pm 150.5$ & $>0.05$ \\
VAT/TAT & $029 \pm 0.15$ & $0.39 \pm 0.13$ & $>0.05$ \\
\hline
\end{tabular}
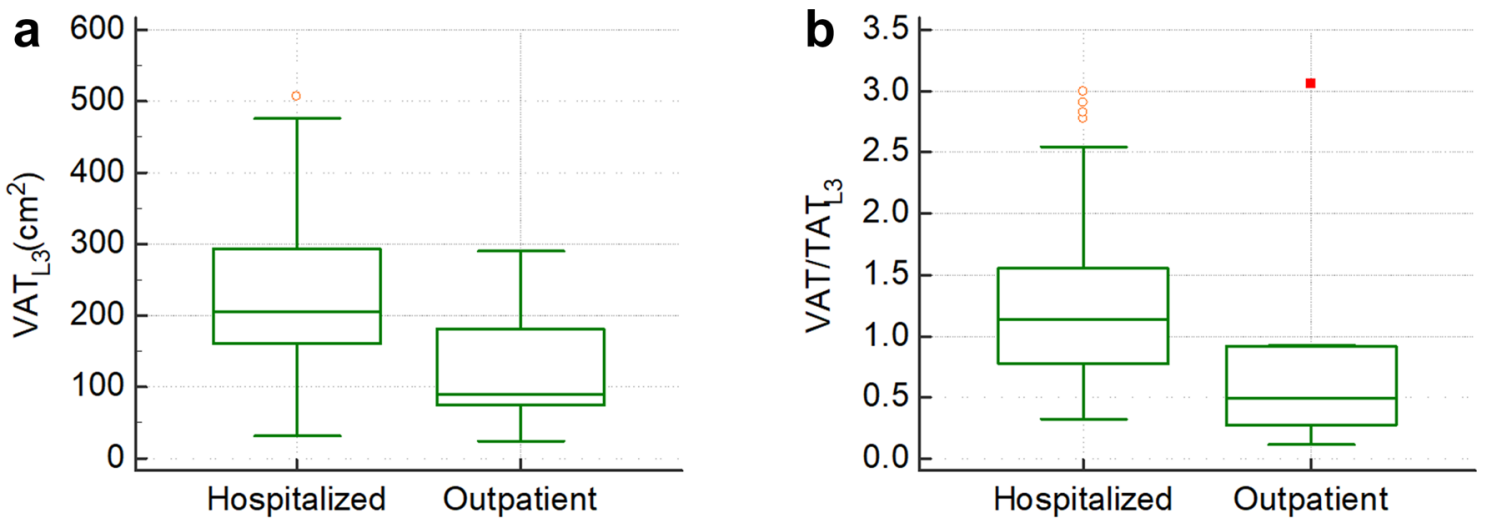

Fig. 3 a $\mathrm{VAT}_{\mathrm{L} 3}$ was significantly higher in hospitalized patients compared to outpatients. b $\mathrm{VAT} \mathrm{TAT}_{\mathrm{L} 3}$ was significantly higher in hospitalized patients compared to outpatients 
Table $4 \mathrm{VAT}_{\mathrm{L} 3}, \mathrm{SAT}_{\mathrm{L} 3}, \mathrm{TAT}_{\mathrm{L} 3}$, and $\mathrm{VAT} \mathrm{TAT}_{\mathrm{L} 3}$ in outpatients and hospitalized men

\begin{tabular}{lccc}
\hline & Outpatient & Hospitalized & $p$ value \\
\hline VAT $\left(\mathrm{cm}^{2}\right)$ & $163.6 \pm 114.0$ & $241.4 \pm 138.5$ & $>0.05$ \\
SAT $\left(\mathrm{cm}^{2}\right)$ & $226.7 \pm 168.2$ & $195.5 \pm 111.0$ & $>0.05$ \\
TAT $\left(\mathrm{cm}^{2}\right)$ & $390.3 \pm 202.7$ & $436.9 \pm 211.0$ & $>0.05$ \\
VAT/TAT & $0.42 \pm 0.23$ & $0.55 \pm 0.18$ & $>0.05$ \\
\hline
\end{tabular}

\section{Correlation of BMI with $\mathrm{VAT}_{\mathrm{L} 3}$ and $\mathrm{SAT}_{\mathrm{L} 3}$}

There was no significant correlation between $\mathrm{VAT}_{\mathrm{L} 3}$ and $\operatorname{BMI}(0.21 ; p=0.16)$, whereas there was a statistically significant strong correlation between $\mathrm{SAT}_{\mathrm{L} 3}$ and $\mathrm{BMI}(0.84$; $p<0.001)$ as well as $\mathrm{TAT}_{\mathrm{L} 3}$ and $\mathrm{BMI}(0.72 ; p<0.001)$. There was inverse correlation between $\mathrm{VAT}^{\mathrm{TAT}} \mathrm{L}_{3}$ and $\mathrm{BMI}$ $(-0.39 ; p=0.006)$.

\section{Repeatability of single-slice measurements}

Intraclass coefficient correlation for $\mathrm{VAT}_{\mathrm{L} 3}, \mathrm{SAT}_{\mathrm{L} 3}$, and $\mathrm{VAT} \mathrm{TAT}_{\mathrm{L} 3}$ was 0.99 for each of these measures. The average difference in $\mathrm{VAT}_{\mathrm{L} 3}$ between the two readers was $1.8 \%$, and it was $1.5 \%$ for $\mathrm{SAT}_{\mathrm{L} 3}$.

\section{Correlation between single-slice and volumetric measures}

In 25 subjects, $\mathrm{VAT}_{\mathrm{L} 3}, \mathrm{SAT}_{\mathrm{L} 3}$, and $\mathrm{VAT}_{/ \mathrm{TAT}_{\mathrm{L} 3}}$ were correlated to volumetric measures. There were statistically strong correlation in $\operatorname{VAT}_{\mathrm{L} 3-3 \mathrm{D}}(0.87 ; p<0.001), \mathrm{SAT}_{\mathrm{L} 3-3 \mathrm{D}}(0.96$; $p<0.001)$, and VAT/TAT $\mathrm{L} 3-3 \mathrm{D}(0.97 ; p<0.001)$.

\section{Models to discriminate hospitalized patients from outpatients}

Clinical (age, sex, BMI) and Clinical plus CT (includes $\mathrm{VAT}_{\mathrm{L} 3}$ ) models were compared to identify patients that required hospitalization. The Clinical $+\mathrm{CT}$ model better fitted the data with BIC of 47.51 compared to the BIC of 50.39 for the clinical model alone. Chi-squared test demonstrated significant $(p=0.009)$ improvement of clinical + CT model compared to the clinical model with value of 6.75 . Area under the curve (AUC) of the clinical + CT was higher compared to the clinical model ( 0.847 versus 0.750$)$ on ROC analysis for identifying patients requiring hospitalization (Fig. 4).

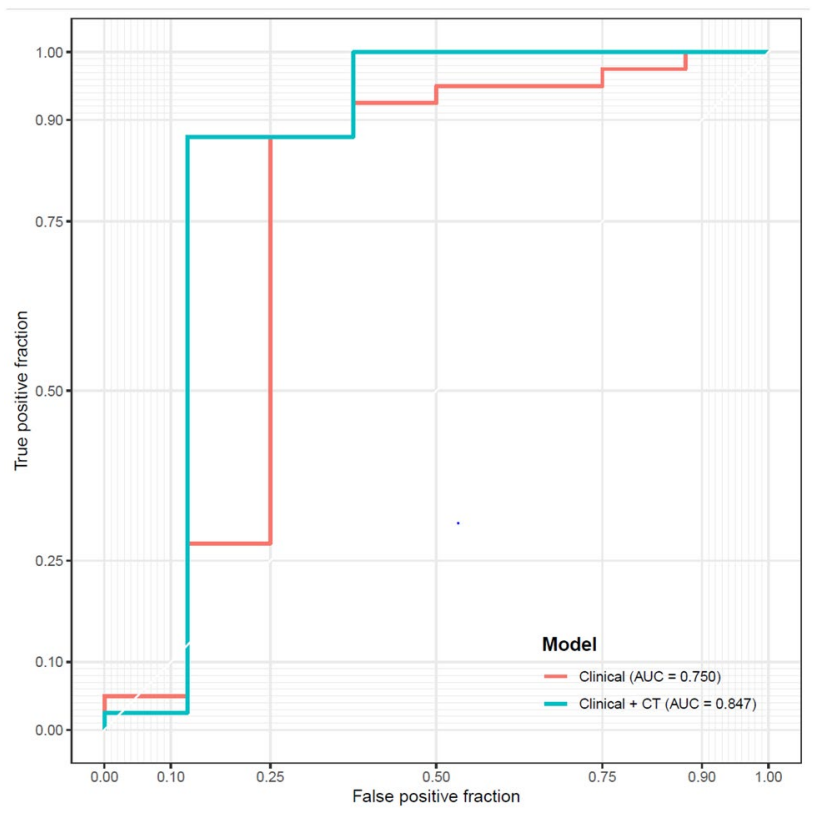

Fig. 4 ROC analysis demonstrates higher AUC of the Clinical+CT model (0.847) compared to the clinical model (0.750) in discriminating patients requiring hospitalization from the outpatients, and this difference was statistically significant

\section{Discussion}

A number of studies have highlighted an association of obesity with severity of COVID-19 symptoms, including hospitalization and need for invasive mechanical ventilation [7, 8]. It is well understood that there are different types of adipose tissue depots that contribute to obesity. These include VAT and SAT, which confer different degree of risks for metabolic disorders and cardiovascular risks [13, 15]. Furthermore, VAT is metabolically more active compared to SAT and is associated with release of pro-inflammatory markers [16]. Hence, in this study we assessed $\mathrm{VAT}_{\mathrm{L} 3}, \mathrm{SAT}_{\mathrm{L} 3}$, and $\mathrm{TAT}_{\mathrm{L} 3}$ on abdominopelvic CT in SARS-CoV-2-positive patients with different severity of COVID-19 infection, and (2) assessed correlations between $\mathrm{BMI}$ and $\mathrm{VAT}_{\mathrm{L} 3}, \mathrm{SAT}_{\mathrm{L} 3}$, $\mathrm{TAT}_{\mathrm{L} 3}$, and $\mathrm{VAT} / \mathrm{TAT}_{\mathrm{L} 3}$ estimates at $\mathrm{CT}$ in patients with COVID-19.

Our results demonstrated significant higher $\mathrm{VAT}_{\mathrm{L} 3}$ and $\mathrm{VAT} \mathrm{TAT}_{\mathrm{L} 3}$ in hospitalized patients compared to the outpatients. Patients admitted to ICU with need for MV had higher $\mathrm{VAT}_{\mathrm{L} 3}$ compared to the hospitalized patients that were not admitted to ICU, but this was not statistically significant. It is important to note that many factors are important in assessing the need for MV including patient's DNR (do not resuscitate) status or availability of the ICU beds. Hence, severity of disease is not the only factor contributing to the ICU admission and MV. This study did not explore these other variables. Men and women have different distribution 
of VAT and SAT, therefore, we stratified our results by gender. In female patients, $\mathrm{VAT}_{\mathrm{L} 3}$ was significantly higher in hospitalized patients compared to outpatients. In men, $\mathrm{VAT}_{\mathrm{L} 3}$ was also higher in hospitalized patients but this did not reach statistical significance.

BMI is a readily available clinical measure of obesity but it does not capture all dimensions of obesity, and adipose tissue distribution may provide additional information not captured in BMI [20-22]. It is interesting to note that BMI demonstrated significantly strong correlation with $\mathrm{SAT}_{\mathrm{L} 3}$ and $\mathrm{TAT}_{\mathrm{L} 3}$, but not with $\mathrm{VAT}_{\mathrm{L} 3}$. Therefore, in these patients $\mathrm{VAT}_{\mathrm{L} 3}$ seems to provide information which is not reflected in BMI. To test the hypothesis that CT assessment of $\mathrm{VAT}_{\mathrm{L} 3}$ provides a clinically important additional information we developed a model that included $\mathrm{VAT}_{\mathrm{L} 3}$ in addition to age, sex, and BMI to discriminate between outpatients and hospitalized patients. The clinical model had AUC of 0.750 . Including $\mathrm{VAT}_{\mathrm{L} 3}$ to this model $($ Clinical $+\mathrm{CT})$ significantly improved the performance of the model in discriminating outpatient from hospitalized patients with AUC of 0.847.

We performed single-slice evaluation of adipose tissue content at the L3 vertebral body level. A number of studies have shown that a single-slice evaluation provides good estimate of the total abdominal fat content [17]. To confirm this hypothesis in COVID-19 patients in our cohort we correlated VAT and SAT measures obtained on a single-slice to volumetric measures in 25 subjects. There was excellent statistically significant correlation between $\mathrm{VAT}_{\mathrm{L3}-3 \mathrm{D}}$, $\mathrm{SAT}_{\mathrm{L3} 3 \mathrm{D}}$, and VAT/TAT $\mathrm{T}_{\mathrm{L3}-3 \mathrm{D}}$. While easier to integrate into clinical practice than volumetric measurements, single-slice workflow is semi-automated as it requires 1 to 2 minutes of user interaction. Our group is exploring an automated workflow solution for volumetric calculation of abdominal fat without any user interaction. Such a workflow could enable routine clinical use of volumetric measure of VAT, SAT, and VAT/TAT.

As in prior studies we also observed that the male gender was associated with hospitalization and ICU admission $[7,23]$ and when comparing outpatients to the hospitalized patients, this difference was statistically significant. Older age was also associated with hospitalization and ICU admission but in this small study these differences were not statistically significant. This was probably related to small sample size of our study.

Other risk factors such as diabetes mellitus, hypertension, history of cardiopulmonary disease, and immunocompromised state have also been identified as risk factors for hospitalization and severe disease [7, 23, 24]. In this small study we did not evaluate these other variables that are also associated with severe disease. This is one of the limitation of our study. Inclusion of these other variables in a larger study may further improve the ability to predict which patients at the time of presentation will require hospitalization. Furthermore, we did not separate intramuscular fat from SAT. The contribution of intramuscular fat can be assessed in the future as it could provide information about sarcopenia which could be an independent risk factor for hospitalization and severe outcomes. In this retrospective study, in some subset of outpatients the patient weight may have been self-reported rather than measured and this could impact BMI calculation.

In conclusion, this study demonstrates higher VAT was observed in COVID-19 patients that required hospitalization compared to the outpatients, and addition of VAT to the clinical model significantly improved its AUC in discriminating hospitalized from outpatients in this small study. More work needs to be done in larger datasets to assess if addition of VAT to clinical models can help stratify patient's need for hospitalization and MV.

Author contributions All authors have fulfilled ICJME criteria for authorship.

Funding This work is supported in part by the National Institute of Health (NIH) Grant U24 EB028980.

Code/software availability Firevoxel software is available upon request.

\section{Compliance with ethical standards}

Conflict of interest Research support not related to current project. Hersh Chandarana: Support in form of hardware and software from Siemens Healthineers.

Ethical approval IRB approved retrospective study.

Informed consent Waiver of consent.

\section{References}

1. Jogalekar MP, Veerabathini A, Gangadaran P. Novel 2019 coronavirus: Genome structure, clinical trials, and outstanding questions. Exp Biol Med (Maywood). 2020:1535370220920540.

2. Zhao S, Lin Q, Ran J, et al. Preliminary estimation of the basic reproduction number of novel coronavirus (2019-nCoV) in China, from 2019 to 2020: A data-driven analysis in the early phase of the outbreak. Int J Infect Dis. 2020;92:214-217.

3. Huang C, Wang Y, Li X, et al. Clinical features of patients infected with 2019 novel coronavirus in Wuhan, China. Lancet. 2020;395(10223):497-506.

4. Chen $\mathrm{G}, \mathrm{Wu} \mathrm{D}, \mathrm{Guo} \mathrm{W}$, et al. Clinical and immunological features of severe and moderate coronavirus disease 2019. J Clin Invest. 2020;130(5):2620-2629.

5. Guan WJ, Ni ZY, Hu Y, et al. Clinical Characteristics of Coronavirus Disease 2019 in China. N Engl J Med. 2020;382(18):1708-1720. 
6. Livingston E, Bucher K. Coronavirus Disease 2019 (COVID-19) in Italy. JAMA. 2020.

7. Petrilli CM, Jones SA, Yang J, et al. Factors associated with hospital admission and critical illness among 5279 people with coronavirus disease 2019 in New York City: prospective cohort study. BMJ. 2020;369:m1966.

8. Kalligeros M, Shehadeh F, Mylona EK, et al. Association of Obesity with Disease Severity among Patients with COVID-19. Obesity (Silver Spring). 2020.

9. Cercato C, Fonseca FA. Cardiovascular risk and obesity. Diabetol Metab Syndr. 2019;11:74.

10. Ellulu MS, Patimah I, Khaza'ai H, Rahmat A, Abed Y. Obesity and inflammation: the linking mechanism and the complications. Arch Med Sci. 2017;13(4):851-863.

11. Vardhana SA, Wolchok JD. The many faces of the anti-COVID immune response. J Exp Med. 2020;217(6).

12. Michalakis K, Ilias I. SARS-CoV-2 infection and obesity: Common inflammatory and metabolic aspects. Diabetes Metab Syndr. 2020;14(4):469-471.

13. Abraham TM, Pedley A, Massaro JM, Hoffmann U, Fox CS. Association between visceral and subcutaneous adipose depots and incident cardiovascular disease risk factors. Circulation. 2015;132(17):1639-1647.

14. Ibrahim MM. Subcutaneous and visceral adipose tissue: structural and functional differences. Obes Rev. 2010;11(1):11-18.

15. Fox CS, Massaro JM, Hoffmann U, et al. Abdominal visceral and subcutaneous adipose tissue compartments: association with metabolic risk factors in the Framingham Heart Study. Circulation. 2007;116(1):39-48.

16. Shuster A, Patlas M, Pinthus JH, Mourtzakis M. The clinical importance of visceral adiposity: a critical review of methods for visceral adipose tissue analysis. Br J Radiol. 2012;85(1009):1-10.
17. Irlbeck T, Massaro JM, Bamberg F, O’Donnell CJ, Hoffmann U, Fox CS. Association between single-slice measurements of visceral and abdominal subcutaneous adipose tissue with volumetric measurements: the Framingham Heart Study. Int J Obes (Lond). 2010;34(4):781-787.

18. Kuk JL, Church TS, Blair SN, Ross R. Does measurement site for visceral and abdominal subcutaneous adipose tissue alter associations with the metabolic syndrome? Diabetes Care. 2006;29(3):679-684.

19. Dane B, Brusca-Augello G, Kim D, Katz DS. Unexpected Findings of Coronavirus Disease (COVID-19) at the Lung Bases on Abdominopelvic CT. AJR Am J Roentgenol. 2020:1-4.

20. Shah RV, Murthy VL, Abbasi SA, et al. Visceral adiposity and the risk of metabolic syndrome across body mass index: the MESA Study. JACC Cardiovasc Imaging. 2014;7(12):1221-1235.

21. Nattenmueller J, Hoegenauer H, Boehm J, et al. CT-based compartmental quantification of adipose tissue versus body metrics in colorectal cancer patients. Eur Radiol. 2016;26(11):4131-4140.

22. Camhi SM, Bray GA, Bouchard C, et al. The relationship of waist circumference and BMI to visceral, subcutaneous, and total body fat: sex and race differences. Obesity (Silver Spring). 2011;19(2):402-408.

23. Cummings MJ, Baldwin MR, Abrams D, et al. Epidemiology, clinical course, and outcomes of critically ill adults with COVID19 in New York City: a prospective cohort study. Lancet. 2020.

24. Richardson S, Hirsch JS, Narasimhan M, et al. Presenting Characteristics, Comorbidities, and Outcomes Among 5700 Patients Hospitalized With COVID-19 in the New York City Area. JAMA. 2020.

Publisher's Note Springer Nature remains neutral with regard to jurisdictional claims in published maps and institutional affiliations. 